\title{
Electrical Conductivity and Modulus Studies of $x$ [CNFO]-(1-x) [0.5BCT-0.5BZT] Multiferroic with Dielectric, Magnetic and Magneto-dielectric Properties
}

\author{
A. B. Kakade, ${ }^{1}$ S. K. Deshpande ${ }^{2}$ and S. B. Kulkarni ${ }^{1, *}$
}

\begin{abstract}
$x\left[\mathrm{CO}_{0.9} \mathrm{Ni}_{0.1} \mathrm{Fe}_{2} \mathrm{O}_{4}\right]-(1-\mathrm{x})\left[0.5 \mathrm{Ba}_{0.7} \mathrm{Ca}_{0.3} \mathrm{TiO}_{3}-0.5 \mathrm{BaZr}_{0.2} \mathrm{Ti}_{0.8} \mathrm{O}_{3}\right]$ multiferroic composite was formed by hydroxide co-precipitation method for $x=0.3,0.4 \&$ 0.5. X-ray diffractometer (XRD) and Scanning Electron Microscopy (SEM) were used for the structural and morphological studies of these composites respectively. The perovskite phase of the ferroelectric material and the spinel phase of the ferrite were confirmed by the XRD spectra. An impedance analyzer was used to study the dielectric properties of the composite. Dielectric constant and tangent loss (Quality factor) variation of the composites were investigated for 100 $\mathrm{Hz}$ to $1 \mathrm{MHz}$ frequency. An increase in magnetocapacitance was observed with the increasing magnetic field up to 1 Tesla. The Magnetodielectric (MD) effect of the composites can be explained based on the contribution of interfacial polarization to the dielectric constant which depends on the magnetic field and variation in the dielectric constant produced by induced stress. The alternating current (Ac) conductivity of the materials which depends on the frequency follows Jonscher's universal power law. These materials show Arrhenius behavior which can be confirmed by temperature-dependent dc conductivity. Modulus studies give strong peaks which shift towards higher frequency with increasing temperature. The saturation magnetization of composites increases as $\mathrm{Co}_{0.9} \mathrm{Ni}_{0.1} \mathrm{Fe}_{2} \mathrm{O}_{4}$ (CNFO) content in the composite increases.
\end{abstract}

Keywords: Multiferroic; Magnetodielectric; Magnetization; Conductivity; Modulus.

Received: 27 March 2021; Revised: 11 Jun 2021; Accepted: 21 June 2021.

Article type: Research article.

\section{Introduction}

Ferromagnetic materials show spontaneous magnetization when placed in the external magnetic field, and are widely used in data storage industries ${ }^{[1]}$ while ferroelectric materials show spontaneous polarization after applying an external electric field, and are widely used in the fabrication of sensors and the actuation industry. ${ }^{[2]}$ Multiferroic (MF) materials captured much obsession because of their future applications in devices like magnetic field sensors, spintronics, switches, actuators, transducers, electronic memory, and multifunctional filters. ${ }^{[3,4]}$ Multiferroic materials exhibit two or more ferroic orders amongst ferromagnetism, ferroelasticity, and ferroelectricity simultaneously at room temperature. ${ }^{[5]}$ The combined effect of ferromagnetism and ferroelectricity indicates that applying either an electric field or a magnetic field can generate electric polarization. ${ }^{[6]}$ However, such an

\footnotetext{
${ }^{1}$ Department of Physics, The Institute of Science, 15, Madam Cama

Road, Mumbai, 400032, India.

${ }^{2}$ UGC-DAE Consortium for Scientific Research, Bhabha Atomic

Research Center, Mumbai 400085, India.

*E-mail: sbk_physics@yahoo.com (S.B. Kulkarni)
}

interesting phenomenon generally occurs at a very low temperature and under the application of a powerful magnetic field for single-phase multiferroics due to smaller values of magnetization and electrical polarization. ${ }^{[7]}$ And hence, for device applications use of multiferroic composites made up of ferrites and ferroelectrics is much attributed. ${ }^{[8,9]}$

Since 1972, intensive research has been carried out in composites of ferroelectric and magnetic phases. ${ }^{[10,11]}$ Some MF materials show coupling between dielectric field and magnetization. ${ }^{[12]}$ Over the last decade, researchers are focusing on the magnetodielectric effect in the multiferroic composite. ${ }^{[13,14]}$ Magnetodielectric effect (MD) is defined as a variation of dielectric constant under an application of a magnetic field. Quantitatively MD effect is shown as MD = $|\varepsilon(\mathrm{H})-\varepsilon(0)| / \varepsilon(0)$. But most of the single-phase multiferroics show this kind of coupling at very low temperatures, which is limited by the lattice structures. ${ }^{[15]}$ For $\mathrm{TbMnO}_{3} \mathrm{~T}$. Kimura et $a l .{ }^{[16]}$ revealed a $10 \% \mathrm{MD}$ ratio after the application of a $6 \mathrm{~T}$ magnetic field and spherical spin order at a temperature of 12 $\mathrm{K}$. At room temperature, a much better MD effect is observed for composites than single-phase materials. ${ }^{[17]}$ Lead-based materials like PZT are widely used ferroelectric materials in 
these composites because of their high dielectric constant, low dielectric loss, and wonderful piezoelectric properties. Recently, the piezoelectric coefficient of $448 \mathrm{pC} / \mathrm{N}, \epsilon_{\mathrm{r}}-2126$ and $\mathrm{Tc} \sim 354{ }^{\circ} \mathrm{C}$ was reported for PZT-Sr(Na $\left.{ }_{0.25} \mathrm{Nb}_{0.75}\right) \mathrm{O}_{3}(\mathrm{PZT}-$ SNN) composite. ${ }^{[18]}$ But due to their toxic nature, there is a need for an alternative for PZT-based materials like $\mathrm{BaTiO}_{3}$, $\mathrm{BiNaTiO}_{3}, \mathrm{KNaNbO}_{3}, \mathrm{BaZrTiO}_{3}(\mathrm{BZT}), \mathrm{BaCaTiO}_{3}(\mathrm{BCT})$ and a solid solution of BCT-BZT is increasing for ferroelectric phase. ${ }^{[19,20]}$ Shi. Su et al. ${ }^{[21]}$ had used the solid-state reaction method to fabricate $0.5 \mathrm{Ba}_{0.7} \mathrm{Ca}_{0.3} \mathrm{TiO}_{3}-0.5 \mathrm{BaZr}_{0.2} \mathrm{Ti}_{0.8} \mathrm{O}_{3}$ and found the highest value of $\mathrm{d}_{33}=630 \mathrm{pC} / \mathrm{N}$ and electrochemical coupling factor of $56 \%$.

$\mathrm{CoFe}_{2} \mathrm{O}_{4}$ is used as a magnetostrictive phase in $\mathrm{ME}$ materials, because of its large values of saturation magnetization, high magnetostriction coefficient $(\lambda s=$ $\left.160 \times 10^{-6}\right)$, and high Curie temperature $(\mathrm{Tc}=247 \mathrm{~K})$. The substitution of $\mathrm{Ni}$ in $\mathrm{CoFe}_{2} \mathrm{O}_{4}$ gives high permeability and a high value of magnetostriction. P. Pan et al. ${ }^{[22]}$ studied magnetodielectric properties of (1$x)\left(\mathrm{Ba}_{0.88} \mathrm{Ca}_{0.12}\right)\left(\mathrm{Ti}_{0.88} \mathrm{Zr}_{0.12}\right) \mathrm{O}_{3}-\mathrm{xCoFe}_{2} \mathrm{O}_{4}$ composite. They have reported the greater value of magnetocapacitance $\mathrm{MD}=$ $5.37 \%$ at $1 \mathrm{kHz}$ frequency and magnetic field of $1.5 \mathrm{~T}$ for $\mathrm{x}=$ 0.4 . Various chemical methods can be used for the synthesis of MF composites like the sol-gel method, ${ }^{[23]}$ co-precipitation method, ${ }^{[24]}$ hydrothermal method, ${ }^{[25]}$ etc.

In this study, MF composite is synthesized by hydroxide co-precipitation method by using $0.5 \mathrm{Ba}_{0.7} \mathrm{Ca}_{0.3} \mathrm{TiO}_{3}-$ $0.5 \mathrm{BaZr}_{0.2} \mathrm{Ti}_{0.8} \mathrm{O}_{3}(0.5 \mathrm{BCT}-0.5 \mathrm{BZT})$ for a ferroelectric phase and $\mathrm{Co}_{0.9} \mathrm{Ni}_{0.1} \mathrm{Fe}_{2} \mathrm{O}_{4}(\mathrm{CNFO})$ for a magnetostrictive phase due to their high piezoelectric coefficient $\left(\mathrm{d}_{33}=630 \mathrm{pC} / \mathrm{N}\right)$ and saturation magnetization $(92.87 \mathrm{emu} / \mathrm{gm})$ respectively. Most of the studies until now were done on the dielectric, magnetic, and MD properties of the given composite. But the electrical properties of these composites are not discussed much in the above-mentioned publications. Therefore dielectric, magnetic and magnetodielectric properties along with electrical properties like conductivity and modulus are studied using different techniques for given composites.

\section{Experimental}

\subsection{Synthesis of BCT, BZT, and 0.5BCT-0.5BZT}

The hydroxide co-precipitation method was used to prepare BCT and BZT separately. The co-precipitation method is a simple, cheap, and environmentally friendly method that requires a lower reaction temperature and short reaction time. For this synthesis, $99.9 \%$ pure $\mathrm{AR}$ graded $\mathrm{Ba}\left(\mathrm{NO}_{3}\right)_{2}$ (barium nitrate), $\mathrm{Ca}\left(\mathrm{NO}_{3}\right)_{2} \cdot 4 \mathrm{H}_{2} \mathrm{O}$ (calcium nitrate), $\mathrm{Zr}\left(\mathrm{NO}_{3}\right) \cdot 2 \mathrm{H}_{2} \mathrm{O}$ (zirconyl nitrate), and $\mathrm{K}_{2} \mathrm{TiO}\left(\mathrm{C}_{2} \mathrm{O}_{2}\right)_{4} \cdot 2 \mathrm{H}_{2} \mathrm{O}$ (potassium titanium oxalate) were taken as precursors. The solution was formed by dissolving these precursors separately in double distilled water and then the whole mixture was washed with distilled water and $\mathrm{NH}_{4} \mathrm{OH}$ 4-5 times to get the precipitate. $\mathrm{KOH}$ and $\mathrm{NH}_{4} \mathrm{OH}$ were used as precipitating agents. By knowing the solubility of barium hydroxide $\left[\mathrm{Ba}(\mathrm{OH})_{2}\right]$ in water $\mathrm{PH}$ of the solution is maintained at $>10$ by washing it with $\mathrm{NH}_{4} \mathrm{OH}$ because it is insoluble in an alkaline medium. Precipitates obtained were dried and ground for 4 hours separately to form powders. Calcination of these powders was done for 6 hours at $1000{ }^{\circ} \mathrm{C}$ to form oxides of BCT and BZT in proper stoichiometry. To form ferroelectric $0.5 \mathrm{BCT}-0.5 \mathrm{BZT}$ stoichiometric amounts of BCT and BZT were rigorously grounded together.

\subsection{Synthesis of CNFO}

Nanoparticles of CNFO were also formed by the same method as elaborated above. Here, $\mathrm{Co}\left(\mathrm{NO}_{3}\right)_{2} \cdot 6 \mathrm{H}_{2} \mathrm{O}$ (cobaltous nitrate), $\mathrm{Ni}\left(\mathrm{NO}_{3}\right)_{2} \cdot 6 \mathrm{H}_{2} \mathrm{O}$ (nickel nitrate), and $\mathrm{Fe}\left(\mathrm{NO}_{3}\right)_{3} \cdot 9 \mathrm{H}_{2} \mathrm{O}$ (ferric nitrate) were used as starting materials. As discussed above this Analytical Reagent (AR) grade materials are also dissolved in double distilled water separately and then the solution is formed by mixing it. This mixture was then washed thoroughly with double distilled water and $\mathrm{NH}_{4} \mathrm{OH}$ 4-5 times to obtain the final precipitate. After drying this precipitate under an IR lamp, agate mortar is used to grind the powder for 5 hours, then this powder formed is calcined at $1000{ }^{\circ} \mathrm{C}$ for 6 hours to get nanoparticles of CNFO.

\subsection{Synthesis of multiferroic $x \quad\left[\mathrm{Co}_{0.9} \mathrm{Ni}_{0.1} \mathrm{Fe}_{2} \mathrm{O}_{4}\right]-(1-x)$ [0.5Ba ${ }_{0.7} \mathrm{Ca}_{0.3} \mathrm{TiO}_{3}-0.5 \mathrm{BaZr}_{0.2} \mathrm{Ti}_{0.8} \mathrm{O}_{3}$ ]}

Stoichiometric proportions of magnetostrictive CNFO and ferroelectric $0.5 \mathrm{BCT}-0.5 \mathrm{BZT}$ were mixed and grounded for 5 hours to form multiferroic $x \quad\left[\mathrm{Co}_{0.9} \mathrm{Ni}_{0.1} \mathrm{Fe}_{2} \mathrm{O}_{4}\right]-(1-\mathrm{x})$ $\left[0.5 \mathrm{Ba}_{0.7} \mathrm{Ca}_{0.3} \mathrm{TiO}_{3}-0.5 \mathrm{BaZr}_{0.2} \mathrm{Ti}_{0.8} \mathrm{O}_{3}\right]$, for $\mathrm{x}=0.3,0.4 \& 0.5$. These powders were then pressed by the hydraulic press by applying 5 tons of pressure to get a disk-like structure with thickness and diameter of $2 \mathrm{~mm}$ and $10 \mathrm{~mm}$ respectively after adding PVA (Polyvinyl Alcohol) as a binder. PVA was further removed by treating the sample at $600{ }^{\circ} \mathrm{C}$ for $30 \mathrm{~min}$. These pellets and individual materials were then finally sintered at $1150{ }^{\circ} \mathrm{C}$ for 6 hours by conventional sintering.

\subsection{Material characterization}

Parent composition and sintered pellets of BCT, BZT, CNFO, and its composites were used for further characterization. The structural properties of materials were studied using a powder X-ray diffractometer (Benchtop PROTO). JEOL (JSMIT300LV) is used for the investigation of the microstructure of the composites through SEM. Dielectric properties of the composites were measured using Wayne Kerr's 6300B model LCR meter of high frequency for $100 \mathrm{~Hz}-1 \mathrm{MHz}$ from room temperature to $230^{\circ} \mathrm{C}$. $\mathrm{C}_{\mathrm{p}}$ and $\mathrm{Q}$ values measured from LCR (Inductance (1), Capacitance (C), and Resistance (R)) meter were used to calculate the dielectric constant $\left(\varepsilon_{\mathrm{r}}\right)$ and loss tangent $(\tan \delta)$ of the composites. MD measurements of the composites were measured up to $10 \mathrm{kOe}$ applied magnetic field. The magnetic characterizations of materials were measured using a vibrating sample magnetometer (VSM).

\section{Results and discussion \\ 3.1 Structural characteristics}




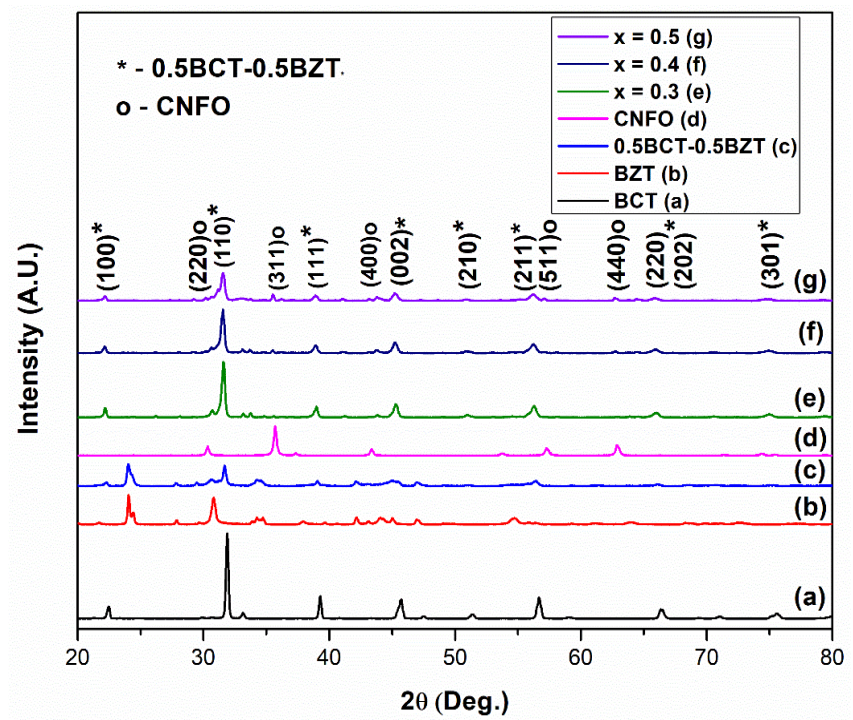

Fig. 1 X-ray diffraction pattern of $\mathrm{x}[\mathrm{CNFO}]-(1-\mathrm{x})$ [0.5BCT$0.5 \mathrm{BZT}]$.

Figure 1 shows $\mathrm{X}$-ray diffraction patterns for BCT, BZT, $\mathrm{CNFO}$, and its composites. Perovskite crystal structure with rhombohedral and tetragonal phases is confirmed by the XRD pattern of 0.5BCT-0.BZT. All the peaks of ferroelectric BZT and BCT tones with the standard JCPDS data with card no. 050626 and no.85-0368. ${ }^{[26]}$ XRD pattern without impurity peaks shows that Calcium and Zirconium are well dissolved in $\mathrm{BaTiO}_{3}$. Cubic spinel structure is observed for CNFO ferrite with $\mathrm{Fd} 3 \mathrm{~m}$ space group. JCPDS card no. 791744 ${ }^{[27]}$ and $742081^{[28]}$ for $\mathrm{CoFe}_{2} \mathrm{O}_{4}$ and $\mathrm{NiFe}_{2} \mathrm{O}_{4}$ confirms the peaks of CNFO. XRD of composites contains all the peaks of CNFO and $0.5 \mathrm{BCT}-0.5 \mathrm{BZT}$. The intensity of peaks of ferrite CNFO increases with an increase in concentration. The debye-
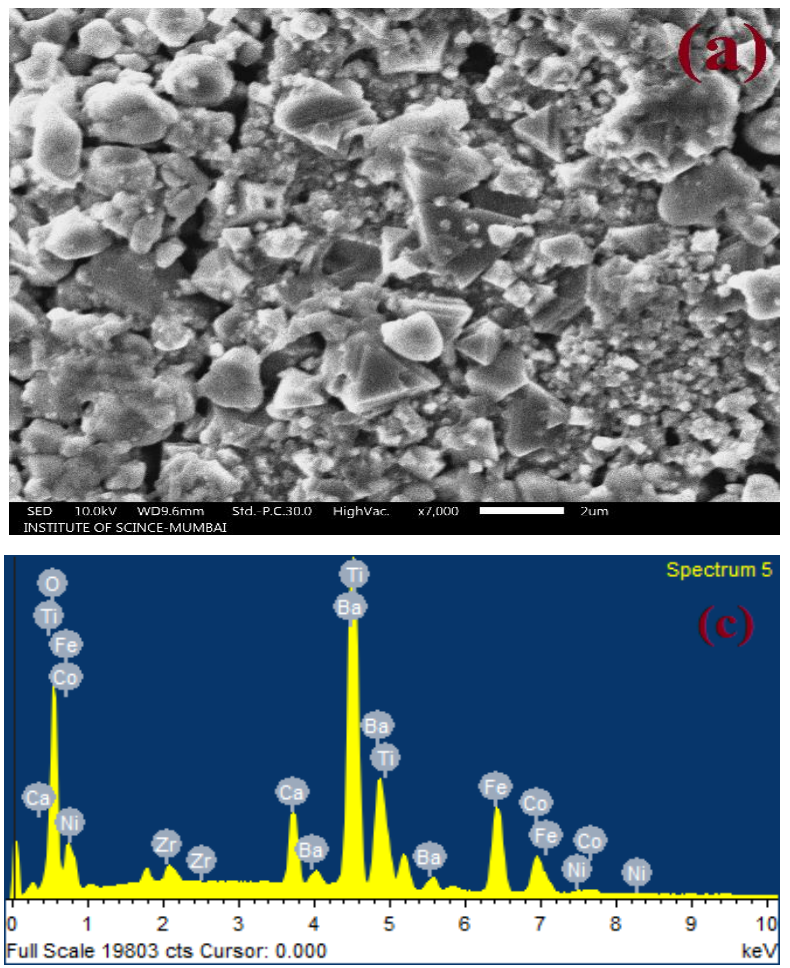

Fig. 2 (a) and (b) SEM images (c) and (d) EDS spectra for $x$

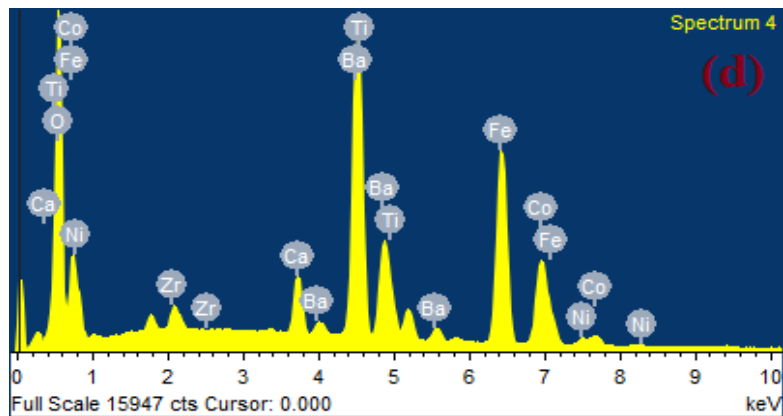

Scherrer equation is used to measure the crystallite size of the materials. ${ }^{[29]}$ Crystallite size for all parent materials and their composites is shown in Table 1 below.

Table 1. Crystallite size of composite $x$ [CNFO]-(1-x) [0.5BCT$0.5 \mathrm{BZT}]$, for $\mathrm{x}=0.3,0.4$ and 0.5 .

\begin{tabular}{lc}
\hline Compositions & Crystallite size $(\mathrm{nm})$ \\
\hline $0.5 \mathrm{BCT}-0.5 \mathrm{BZT}$ & 32.60 \\
CNFO & 63.23 \\
$0.3 \mathrm{CNFO}-0.7(0.5 \mathrm{BCT}-0.5 \mathrm{BZT})$ & 45.07 \\
$0.4 \mathrm{CNFO}-0.6(0.5 \mathrm{BCT}-0.5 \mathrm{BZT})$ & 53.46 \\
$0.5 \mathrm{CNFO}-0.5(0.5 \mathrm{BCT}-0.5 \mathrm{BZT})$ & 55.39 \\
\hline
\end{tabular}

\subsection{SEM analysis (microstructural)}

Micrographs for multiferroic composite $\mathrm{x}$ [CNFO]-(1-x) [0.5BCT-0.5BZT] for $x=0.3$ and 0.5 are shown in Figs. 2(a) and (b) respectively. These micrographs show that smaller and larger grains of BCT-BZT and CNFO respectively are well dispersed in the composite with small agglomeration i.e. they are not well separated from each other. It shows a dense structure without any pores. With increasing ferrite concentration grain size of the composite varies as the concentration of ferroelectric material is more as compared to ferrite in the composite. CNFO grains are distributed well over the grains of BCT-BZT. These distinguishable phases with well compact microstructure confirms formation of composite. Energy dispersive spectroscopy (EDS) study confirms the elemental composition of all composites. EDS spectra of composites for $\mathrm{x}=0.3$ and 0.5 is shown in Figs. 2(c) and (d), respectively. EDS of all composites shows that all elements are present in composites without any impurity.

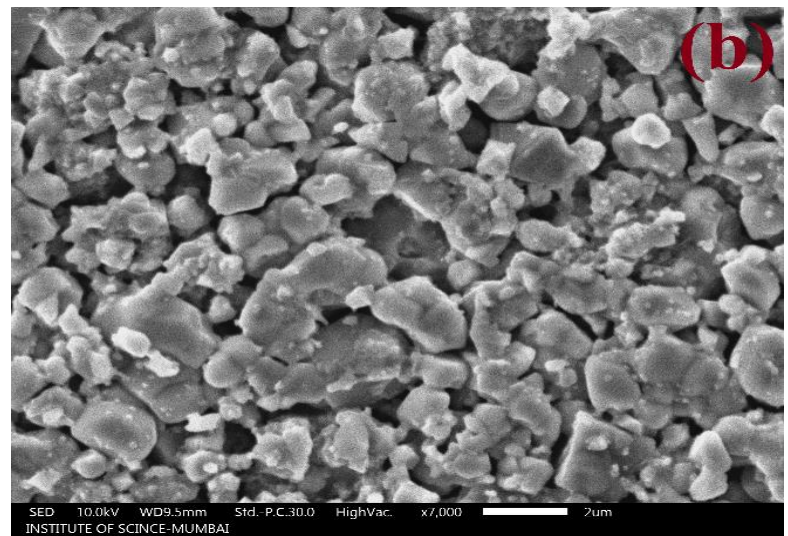

CNFO]-(1-x) [0.5BCT-0.5BZT], $\mathrm{x}=0.3$ and 0.5 respectively. 


\subsection{Dielectric properties}

Frequency-dependent dielectric constant $(\varepsilon)$ and dielectric tangent loss $(\tan \delta)$ for all composites are shown in Fig. 3. From the above figures, it is observed that all the composites of $0.5 \mathrm{BCT}-0.5 \mathrm{BZT}$ and CNFO give higher values for $\varepsilon$. With increasing frequency dielectric constant of the materials decreases. At lower frequencies, $\varepsilon$ values observed are high due to more contribution of space charge polarization. Direct entry of ferrite ions into the ferroelectric site causes disorder
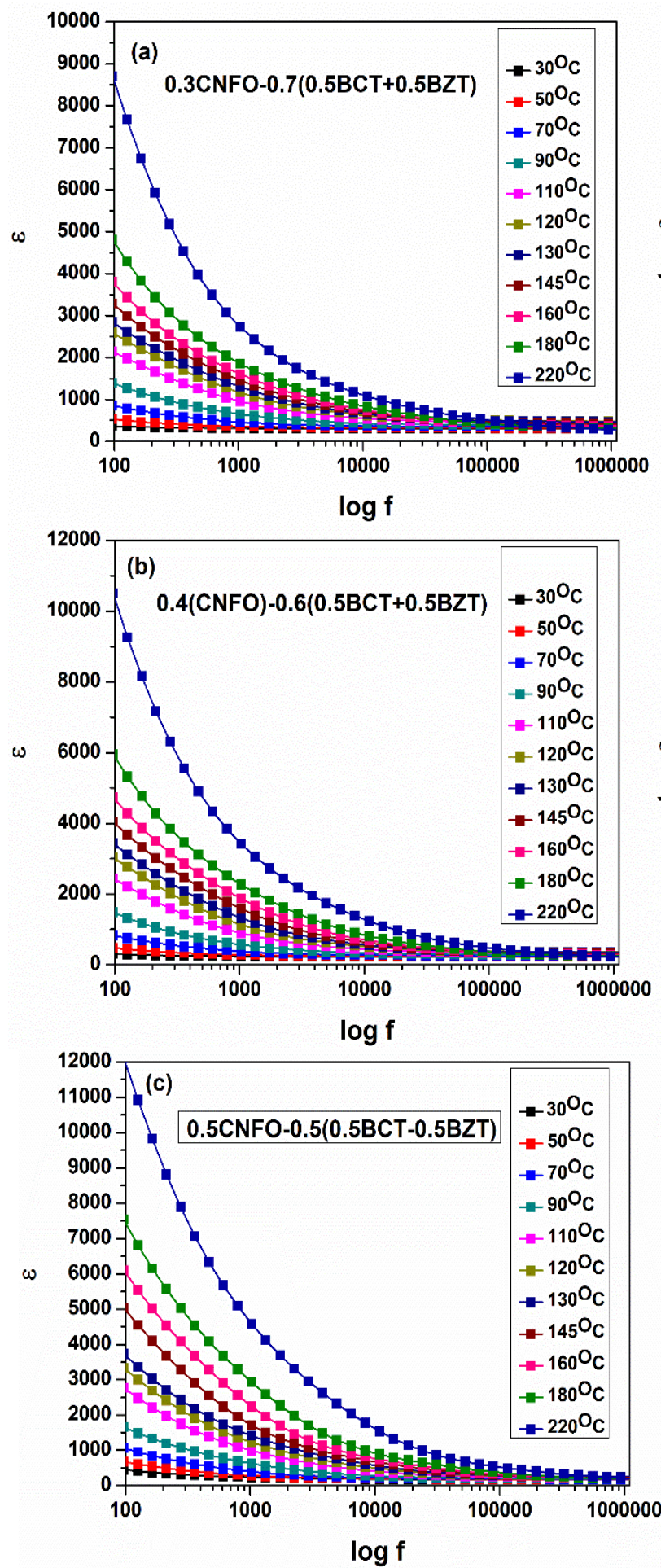

in the arrangement of ferroelectric ions, as a result of which $\varepsilon$ decreases rapidly in this region. At higher frequencies, the decrease in $\varepsilon$ is because electron hopping does not follow any change of electric field beyond a certain frequency range. The loss tangent of the material shows the same behavior as that of $\varepsilon$. With increasing, the frequency loss tangent of the materials also decreases. Both $\varepsilon$ and $\tan \delta$ increase as the ferrite concentration of the composite increases for a given frequency range.
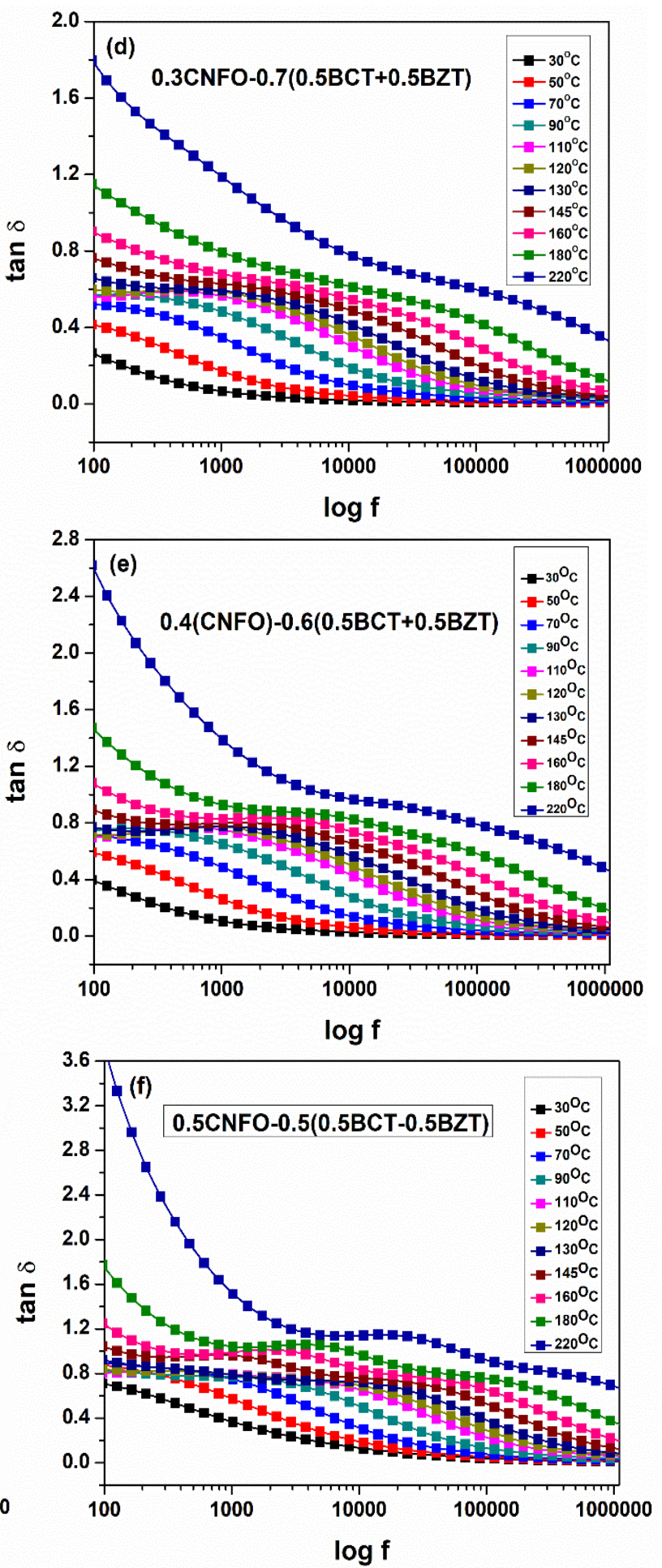

Fig. 3 (a), (b), and (c) Frequency-dependent dielectric constant and (d), (e) and (f) loss tangent for x[CNFO]-(1-x)[0.5BCT-0.5BZT], for $\mathrm{x}=0.3,0.4$ and 0.5 respectively. 
Variations of dielectric constant $(\varepsilon)$ and tangent loss $(\tan \delta)$ with temperature for all composites are shown in Fig. 4. It can be seen from the figure that for each composite $\varepsilon$ increases with an increase in temperature because at higher temperatures conduction takes place. This conduction in the ferrite phase occurs due to the hopping of electrons and holes. With increasing frequency dielectric constant of the material decreases. Different types of frequency-dependent polarization contribute to the dielectric constant of the material. At lower frequencies, interfacial and dipolar polarization contributes while electronic polarization plays
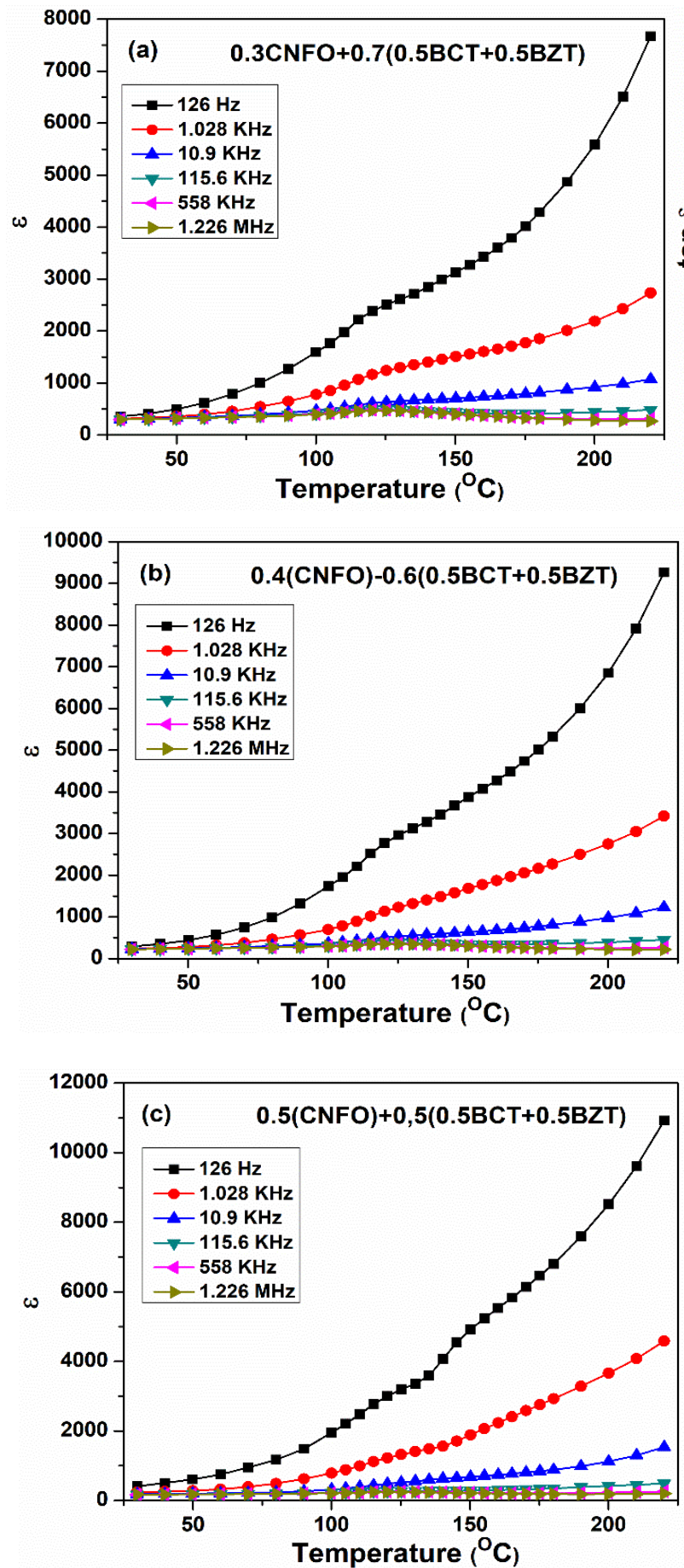

important role in contributing to the dielectric constant at higher frequencies. The loss tangent of the material shows the same behavior as that of $\varepsilon$. Value of $\tan \delta$ increases with increasing frequency for each composite. With increasing frequency $\tan \delta$ shifts towards higher temperatures which shows the relaxer behavior for the given composites. Up to a certain temperature value of $\tan \delta$ remains constant, after which a rapid increase in its value is observed due to increasing hopping electrons at high temperatures space charge conduction takes place.
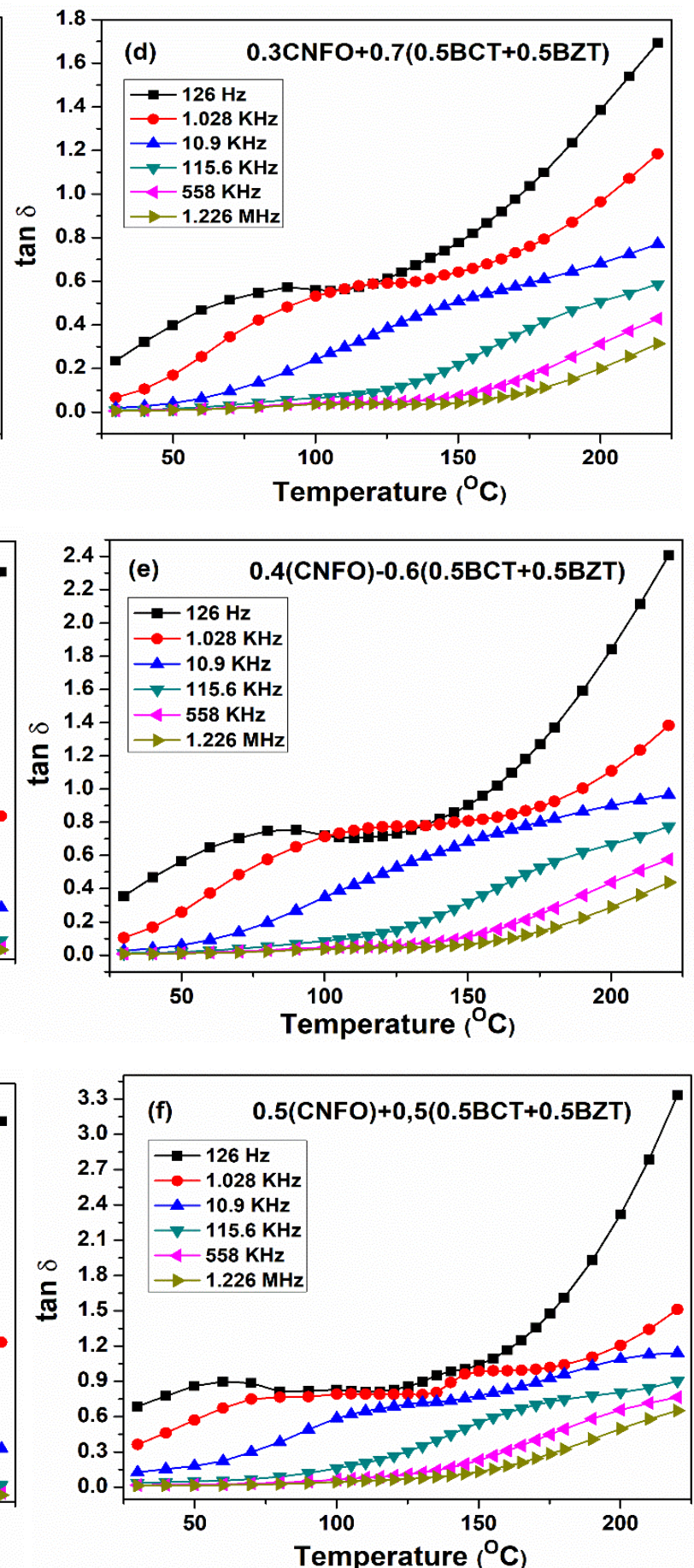

Fig. 4 (a), (b) and (c) Temperature-dependent dielectric constant and (d), (e) and (f) loss tangent for x[CNFO]-(1-x)[0.5BCT-0.5BZT], for $\mathrm{x}=0.3,0.4$ and 0.5 respectively. 


\subsection{Electrical conductivity}

Frequency-dependent ac conductivity of composites of CNFO and $0.5 \mathrm{BCT}-0.5 \mathrm{BZT}$ at $200^{\circ} \mathrm{C}$ is shown in Fig. 5. Variation of electrical transport properties with frequency can be studied with the help of the ac conductivity of materials. It is explained based on Jonscher's power law. ${ }^{[30]}$ This law states that mobile charge carriers provide relaxation to material which then jumps to a new site from its original position, they will remain in between the minima of two potential energy. Frequency independent plateau is seen in the conductivity curve at lower frequencies whereas $\sigma_{\mathrm{ac}}$ is directly proportional to the angular frequency at higher frequencies. As CNFO content in composite increases value of $\sigma_{\mathrm{ac}}$ increases. Increasing conductivity with rising frequency is due to cations disordering and space charge polarization. ${ }^{[31]}$

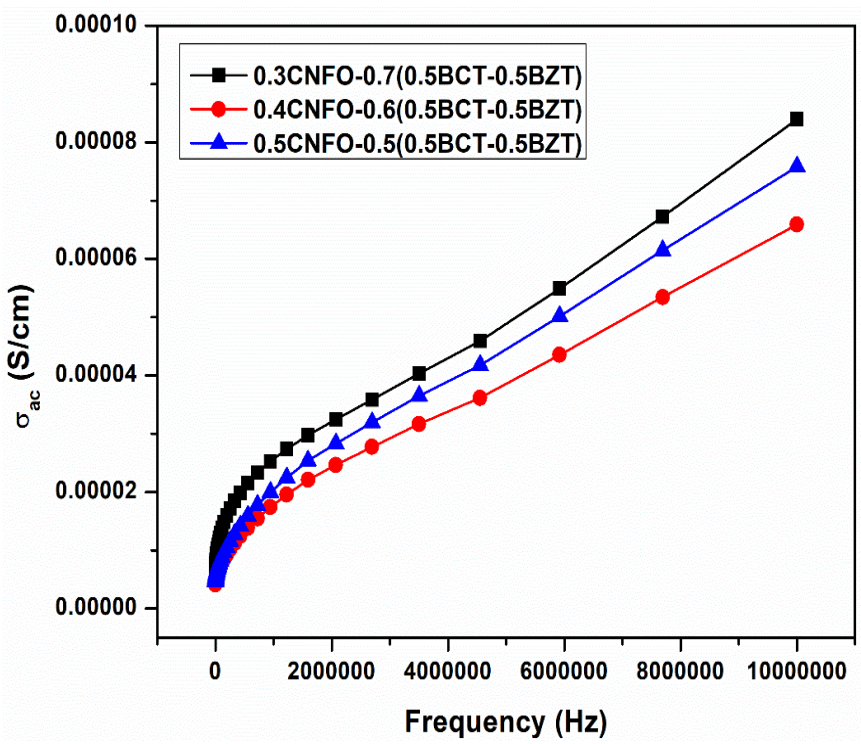

Fig. 5 Frequency-dependent variation of ac conductivity $\left(\sigma_{\mathrm{ac}}\right)$ for $\mathrm{x}[\mathrm{CNFO}]-(1-\mathrm{x})[0.5 \mathrm{BCT}-0.5 \mathrm{BZT}]$ at $200{ }^{\circ} \mathrm{C}$ for $\mathrm{x}=0.3,0.4$, and 0.5 .

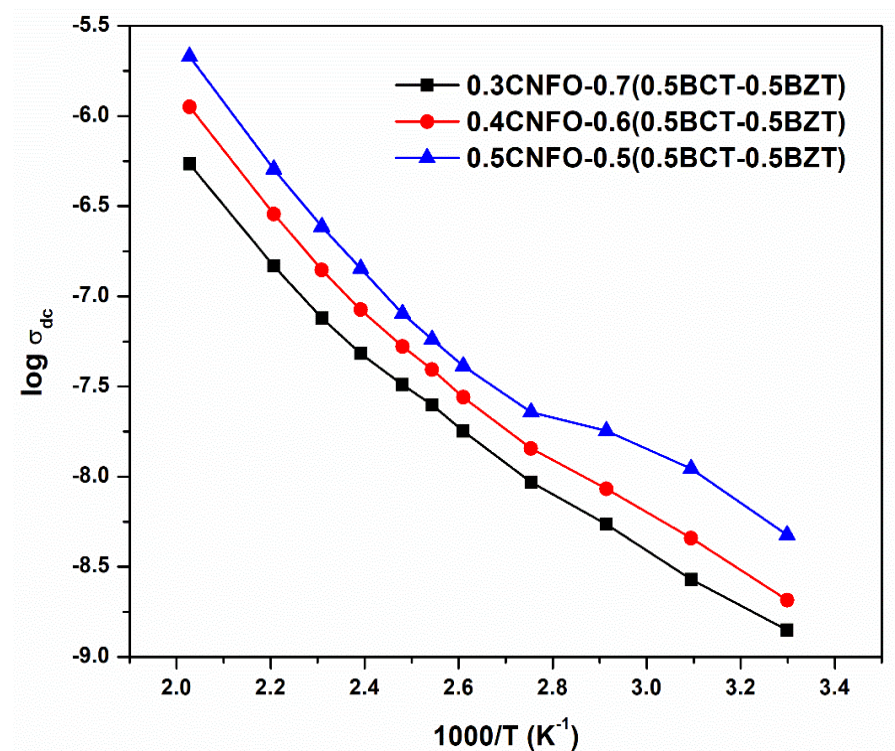

Fig. 6 Variation of dc conductivity with Temperature for $\mathrm{x}$ [CNFO]-(1-x) [0.5BCT-0.5BZT] for $\mathrm{x}=0.3,0.4$ and 0.5 .
Temperature-dependent dc conductivity (i.e. $\sigma_{\mathrm{dc}} \mathrm{Vs} 1000 / \mathrm{T}$ ) of the composite is shown in Fig. 6. The plot follows the Arrhenius behavior. ${ }^{[32]}$ It is observed from the figure that with increasing temperature $\sigma_{\mathrm{dc}}$ increases. NTCR (negative temperature coefficient of resistance) behavior of the composites is validated by dc conductivity studies. ${ }^{[33]}$ Oxygen vacancies in composites produce hopping of charge carriers which causes to increase in the conductivity of composites with an increase in CNFO content. ${ }^{[34]}$ The activation energies calculated for composites are $0.36,0.42$, and $0.44 \mathrm{eV}$ for $\mathrm{x}=$ $0.3,0.4$, and 0.5 respectively.

\section{5 Modulus studies and relaxation time}

Frequency-dependent modulus studies of composites for $\mathrm{x}=$ 0.3, 0.4, and 0.5 at different temperatures are shown in Figs. 7(a)-(c) respectively. The figure shows strong peaks of modulus. Shifting of these peaks towards higher frequencies is observed with increasing temperature. Charge carriers move to longer distances at the frequency under maxima of modulus while at frequencies beyond maxima these charge carriers move on shorter distances because it is restricted by potential well. The peaks observed are asymmetric can be seen from the figure. In the frequency range, where a maximum modulus peak falls gives the transition of mobility from a long distance to a short distance.

Temperature-dependent relaxation time for the composites is shown in Fig. 7(d). From the figure, it is observed that all the curves of the composites follow Arrhenius's behavior. The relaxation values of composites decrease with an increase in temperature for $\mathrm{x}[\mathrm{CNFO}]-(1-\mathrm{x})[0.5 \mathrm{BCT}-0.5 \mathrm{BZT}]$ for $\mathrm{x}=0.3$, 0.4 , and 0.5 . This shows behavior like semiconductors. During high-temperature sintering loss of oxygen causes the semiconductor nature of the grains. Activation energy decreases as oxygen vacancies increases in these types of composites. ${ }^{[35]}$

\subsection{Magnetodielectric properties}

The effect of magnetic field on magnetocapacitance (MC) for composites at frequency $5 \mathrm{kHz}$ and field of and for field 0 $10 \mathrm{kOe}$ is shown in Fig. 8. Increase in magnetocapacitance is observed with increasing CNFO content in composites. It is also observed from the figure that as the magnetic field applied increases for 0-10kOe MC values for composites also increase. The applied magnetic field gets dropped across the boundaries of composites because of more effectiveness of boundaries than that of the grains of composites and hence the capacitance of the composites increases. The composite material gets strained because of the applied magnetic field which further produces stress to give the electric field and dielectric constant of the composite changes. Here in composites, the ferroelectric phase gets oriented because of this electric field.

\subsection{Magnetic properties}

Figure 9 shows the M-H hysteresis loop for CNFO and its all composites at $300 \mathrm{~K}$. VSM is used to measure magnetization 

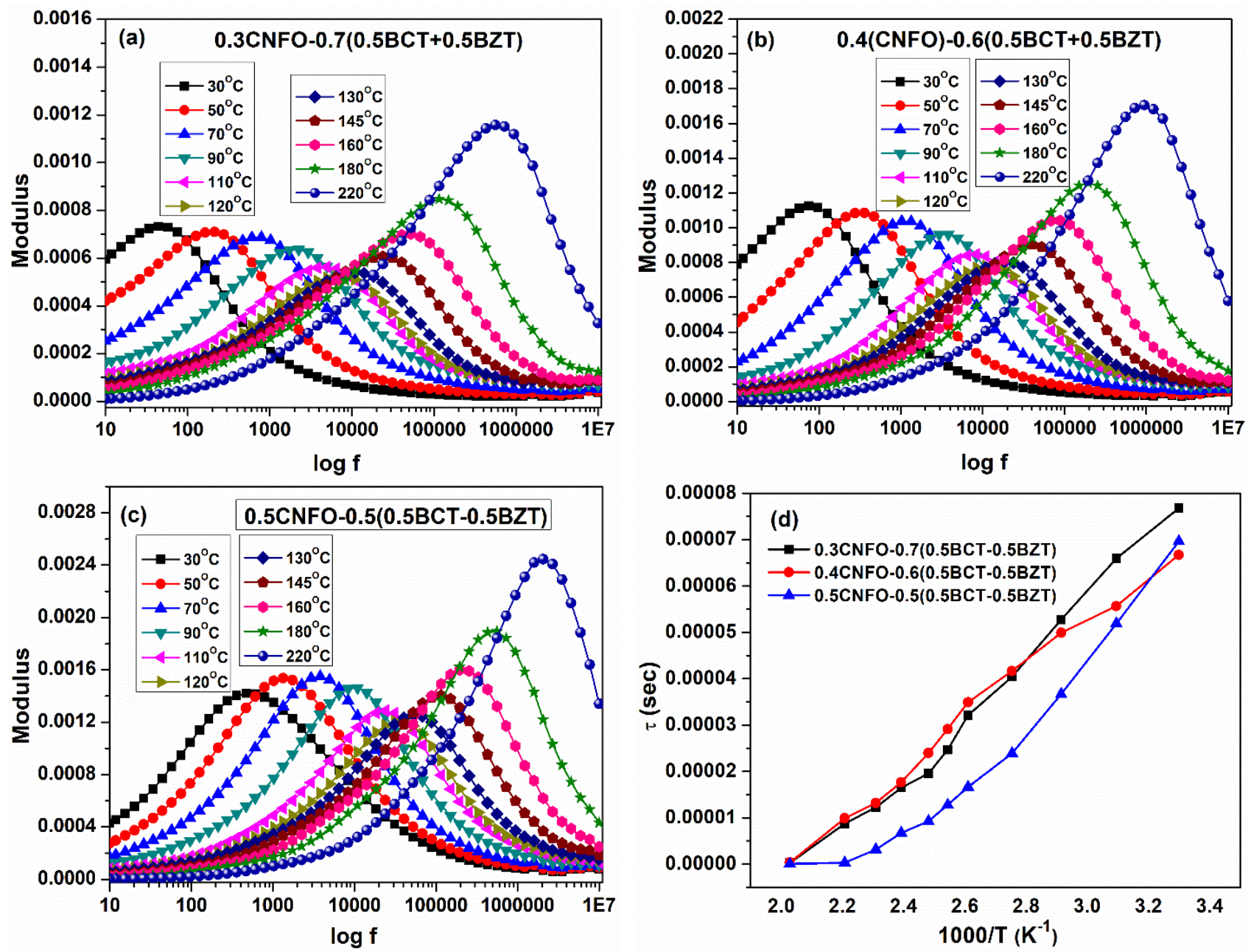

Fig. 7 (a)-(c) Variation of modulus with frequency at different temperatures and (d) variation of relaxation time with temperature.

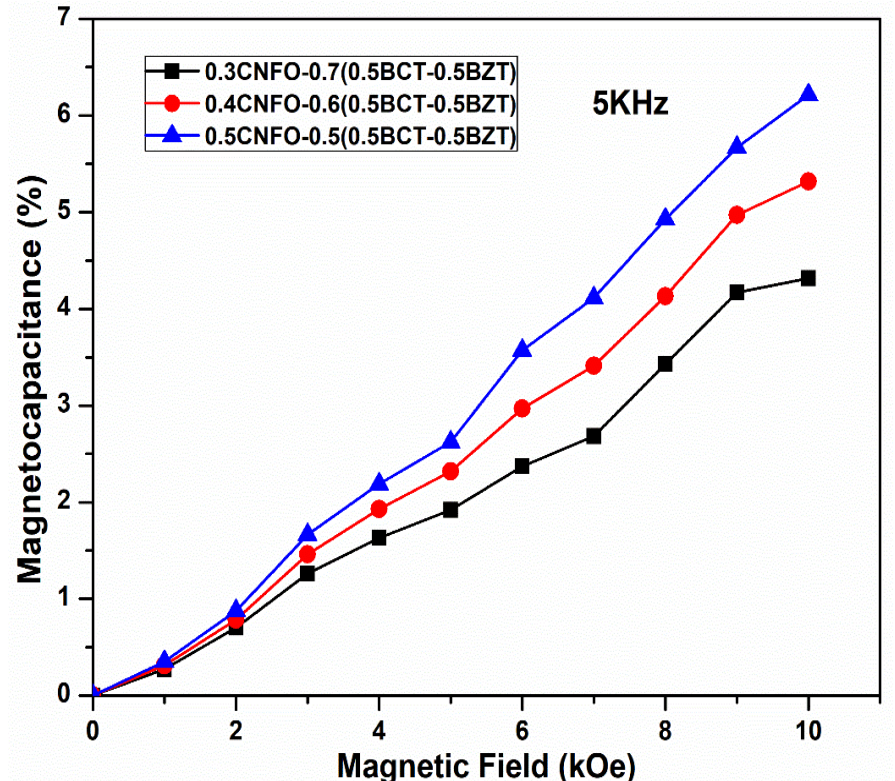

Fig. 8 Variation of magnetocapacitance under applied magnetic field of $x$ [CNFO]-(1-x) [0.5BCT-0.5BZT], $x=0.3,0.4$ and 0.5 at $5 \mathrm{KHz}$.

in the field of range $-90 \mathrm{kOe}$ to $90 \mathrm{kOe}$. Proper curves confirm the ordered magnetic structures of the composites. CNFO shows larger values of remanent $\left(\mathrm{M}_{\mathrm{r}}\right)$ and saturation magnetization $\left(\mathrm{M}_{\mathrm{s}}\right)$ as compared to composites. Easy magnetization of the composites can be indicated by the smaller values of the coercive field $\left(\mathrm{H}_{\mathrm{c}}\right)$. As CNFO concentration increases $M_{s}$ and $H_{c}$ values for composites increases. This increase in CNFO content decreases the pores created by ferroelectric grains. Since more CNFO particles are distributed over the ferroelectric phase as CNFO content increases in composites $\mathrm{H}_{\mathrm{c}}$ values increases. $\mathrm{M}_{\mathrm{s}}, \mathrm{M}_{\mathrm{r}}$, and $\mathrm{H}_{\mathrm{c}}$ for composites are given in Table 2.

Table 2. Values of $\mathrm{M}_{\mathrm{s}}, \mathrm{M}_{\mathrm{r}}$ and $\mathrm{H}_{\mathrm{c}}$ for $\mathrm{x}[\mathrm{CNFO}]-(1-\mathrm{x})[0.5 \mathrm{BCT}$ $0.5 \mathrm{BZT}], \mathrm{x}=0.3,0.4$ and 0.5 at $300 \mathrm{~K}$.

\begin{tabular}{|c|c|c|c|}
\hline Composition & $\mathrm{M}_{\mathrm{s}}[\mathrm{emu} / \mathrm{g}]$ & $\mathrm{M}_{\mathrm{r}}[\mathrm{emu} / \mathrm{g}]$ & $\mathrm{H}_{\mathrm{c}}[\mathrm{Oe}]$ \\
\hline $\mathrm{Co}_{0.9} \mathrm{Ni}_{0.1} \mathrm{Fe}_{2} \mathrm{O}_{4}$ & 86 & 26.31 & 840 \\
\hline $\begin{array}{l}0.3 \mathrm{CNFO}-0.7(0.5 \mathrm{BCT}- \\
0.5 \mathrm{BZT})\end{array}$ & 27.02 & 6.99 & 127 \\
\hline $\begin{array}{l}0.4 \mathrm{CNFO}-0.6(0.5 \mathrm{BCT}- \\
0.5 \mathrm{BZT})\end{array}$ & 34.39 & 4.43 & 190 \\
\hline $\begin{array}{l}0.5 \mathrm{CNFO}-0.5(0.5 \mathrm{BCT}- \\
0.5 \mathrm{BZT})\end{array}$ & 38.34 & 1.52 & 249 \\
\hline
\end{tabular}




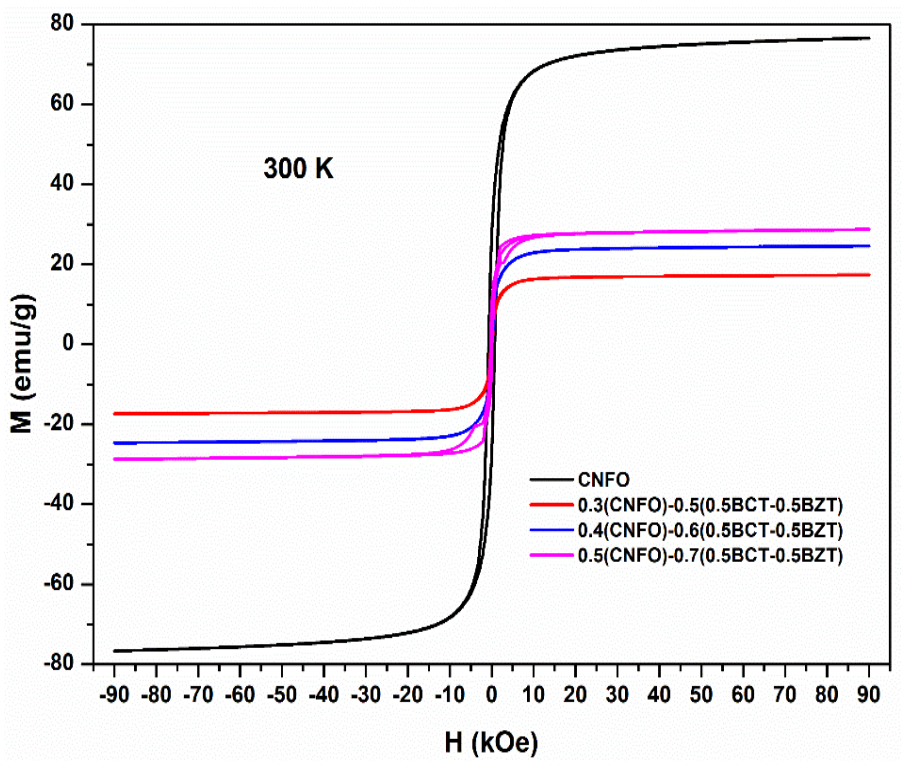

Fig. $9 \mathrm{M}-\mathrm{H}$ hysteresis loop of CNFO and $\mathrm{x}$ [CNFO]-(1-x) [0.5BCT-0.5BZT], $\mathrm{x}=0.3,0.4$ and 0.5 at $300 \mathrm{~K}$.

\section{Conclusion}

Multiferroic composite $\quad x \quad\left[\mathrm{Co}_{0.9} \mathrm{Ni}_{0.1} \mathrm{Fe}_{2} \mathrm{O}_{4}\right]-(1-\mathrm{x})$ $\left[0.5 \mathrm{Ba}_{0.7} \mathrm{Ca}_{0.3} \mathrm{TiO}_{3}-0.5 \mathrm{BaZr}_{0.2} \mathrm{Ti}_{0.8} \mathrm{O}_{3}\right]$ is successfully prepared by co-precipitation method. Its structural, morphological, electrical and magnetic properties studied. XRD pattern confirms the perovskite and spinel structure of ferroelectric and ferrite phases respectively. SEM micrographs shown well dispersed particles of ferrite and ferroelectric phase. Dielectric constant and loss tangent both values increases with ferrite concentration increase while it decreases with increasing frequency. Both values also increases due to electron hopping at higher temperatures with increasing temperature. Relaxer behavior of materials is observed due to shifting of peaks. As the ferrite concentration in composites increases $\mathrm{M}_{\mathrm{s}}$ and $\mathrm{H}_{\mathrm{c}}$ values increases. Variation of ac conductivity of composites with frequency follows Jonscher's power law. NTCR behavior of materials is observed.

\section{Acknowledgement}

The authors are thankful to UGC-DAE CSR for their financial support through project. (Project no. CRS-M-283).

\section{Supporting information}

Applicable.

\section{Conflict of interest}

There are no conflicts to declare.

\section{References}

[1] R. L. Comstock, Journal of Materials Science: Materials in Electronics, 2002, 13, 509-523, doi: 10.1023/A:1019642215245.

[2] K. F. Wang, J. M. Liu, Z. F. Ren, Advances in Physics, 2009, 58, 321-448, doi: 10.1080/00018730902920554.

[3] C. W. Nan, M. I. Bichurin, S. Dong, D. Viehland, G. Srinivasan, Journal of Applied Physics, 2008, 103, 031101, doi:
$10.1063 / 1.2836410$

[4] S. Dong, J. M. Liu, S. W. Cheong, Z. Ren, Advances in Physics, 2015, 64, 519-626, doi: 10.1080/00018732.2015.1114338.

[5] L. W. Martin, S. P. Crane, Y. H. Chu, M. B. Holcomb, M. Gajek, M. Huijben, C. H. Yang, N. Balke, R. Ramesh, Journal of Physics: Condensed Matter, 2008, 20, 434220, doi: 10.1088/0953-8984/20/43/434220.

[6] B. K. Bammannavar, L. R. Naik, Smart Materials and Structures, 2009, 18, 085013, doi: 10.1088/09641726/18/8/085013.

[7] S. W. Cheong, M. Mostovoy, Nature Materials, 2007, 6, 1320, doi: $10.1038 /$ nmat 1804 .

[8] R. Ramesh, N. A. Spaldin, Nature Materials, 2007, 6, 21-29, doi: $10.1038 /$ nmat1805.

[9] S. Y. Tan, S. R. Shannigrahi, S. H. Tan, F. E. H. Tay, Journal of Applied Physics, 2008, 103, 094105, doi: 10.1063/1.2917394.

[10] R. Rani, S. Singh, J. K. Juneja, K. K. Raina, C. Prakash, Ferroelectrics Letters Section, 2011, 38, 134-140, doi: 10.1080/07315171.2011.623612.

[11] X. H. Liu, Z. Xu, X. Y. Wei, X. Yao, Journal of Materials Research, 2010, 25, 1812-1816, doi: 10.1557/JMR.2010.0231.

[12] G. Lawes, A. P. Ramirez, C. M. Varma, M. A. Subramanian, Physical Review Letters, 2003, 91, 257208, doi: 10.1103/PhysRevLett.91.257208.

[13] N. Adhalkha, K. L. Yadav, R. Singh, Smart Materials and Structures, 2014, 23, 105024, 10.1088/0964-1726/23/10/105024.

[14] J. Lee, J. Lee, K. Min, Y. Cheon, IEEE Antennas and Wireless Propagation Letters, 2014, 13, 935-938, doi: 10.1109/lawp.2014.2323066.

[15] Y. Tokura, S. Seki, Advanced Materials, 2010, 22, 15541565, doi: 10.1002/adma.200901961.

[16] T. Kimura, T. Goto, H. Shintani, K. Ishizaka, T. Arima, Y. Tokura, Nature, 2003, 426, 55-58, doi: 10.1038/nature02018.

[17] N. Mufti, A. A. Nugroho, G. R. Blake, T. M. Palstra, Journal of Physics: Condensed Matter, 2010, 22, 075902, doi: 10.1088/0953-8984/22/7/075902.

[18] F. Zhu, J. Qiu, H. Ji, K. Zhu, K. Wen, Journal of Materials Science: Materials in Electronics, 2015, 26, 2897-2904, doi: 10.1007/s10854-015-2775-z.

[19] J. P. Praveen, T. Karthik, A. R. James, E. Chandrakala, S. Asthana, D. Das, Journal of the European Ceramic Society, 2015, 35, 1785-1798, doi: 10.1016/j.jeurceramsoc.2014.12.010.

[20] S. M. Mane, P. M. Tirmali, D. J. Salunkhe, P. B. Joshi, C. B. Kolekar, S. B. Kulkarni, Journal of Materials Science: Materials in Electronics, 2016, 27, 7204-7210, doi: 10.1007/s10854-0164685-0.

[21] S. Su, R. Zuo, S. Lu, Z. Xu, X. Wang, L. Li, Current Applied Physics, 2011, 11, S120-S123, doi: 10.1016/j.cap.2011.01.034.

[22] P. Pan, J. Tao, F. Ma, N. Zhang, Journal of Magnetism and Magnetic Materials, 2018, 453, 91-95, doi: 10.1016/j.jmmm.2017.12.107.

[23] M. M. Sutar, A. N. Tarale, S. R. Jigajeni, S. B. Kulkarni, V. R. Reddy, P. B. Joshi, Solid State Sciences, 2012, 14, 1064-1070, doi: 10.1016/j.solidstatesciences.2012.05.016. 
[24] S. Mane, P. Tirmali, S. Kadam, A. Tarale, C. Kolekar, S. Kulkarni, Journal of the Chinese Advanced Materials Society, 2016, 4, 269-284, doi: 10.1080/22243682.2016.1214924.

[25] S. G. Chavan, S. M. Mane, S. B. Kulkarni, M. E. Jayasingh, P. B. Joshi, D. J. Salunkhe, Journal of Materials Science: Materials in Electronics, 2016, 27, 7105-7108, doi: 10.1007/s10854-016-4672-5.

[26] V. S. Puli, D. K. Pradhan, D. B. Chrisey, M. Tomozawa, G. L. Sharma, J. F. Scott, R. S. Katiyar, Journal of Materials Science, 2013, 48, 2151-2157, doi: 10.1007/s10853-012-6990-1.

[27] A. Kumar, P. Sharma, D. Varshney, Ceramics International, 2014, 40, 12855-12860, doi: 10.1016/j.ceramint.2014.04.140.

[28] K. Maaz, W. Khalid, A. Mumtaz, S. K. Hasanain, J. Liu, J. L. Duan, Physica E: Low-Dimensional Systems and Nanostructures, 2009, 41, 593-599, doi: 10.1016/j.physe.2008.10.009.

[29] A. O. Bokuniaeva, A. S. Vorokh, Journal of Physics: Conference Series, 2019, 1410, 012057, doi: 10.1088/17426596/1410/1/012057.

[30] J. Plcharski, W. Wieczoreck, Solid State Ionics, 1982, 28-30, 979-982, doi: 10.1016/0167-2738(88)90315-3.

[31] A. Shukla, R. N. P. Choudhary, Journal of Materials Science: Materials in Electronics, 2011, 22, 1222, doi: 10.1007/s10854011-0289-x.

[32] J. Rossmacdonald, Solid State Ionics, 1984, 13, 147-149, doi: 10.1016/0167-2738(84)90049-3.

[33] S. Pattanayak, R. N. P. Choudhary, P. R. Das, Journal of Materials Science: Materials in Electronics, 2013, 24, 2767, doi: 10.1007/s10854-013-1168-4.

[34] C. Ang, Z. Yu, L. E. Cross, Physical Review Journals, 2000, 62, 228, doi: 10.1103/PhysRevB.62.228.

[35] J. F. Scott, M. Dawber, Applied Physics Letters, 2000, 76, 3801-3803, doi: 10.1063/1.126786.

\section{Author information}

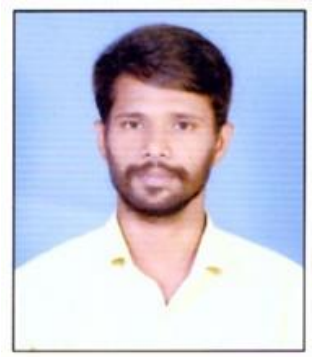

A. B. Kakade is a research student in Department of Physics, The Institute of Science, Mumbai, Maharashtra, India. He has completed his M.Sc. in physics in 2016 from The Institute of Science, Mumbai, Maharashtra. He is now working on multiferroic materials.

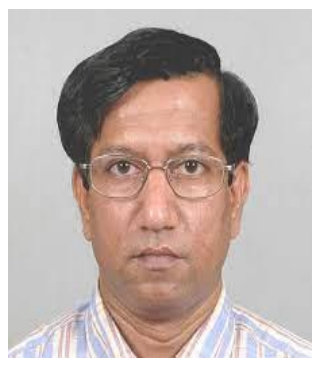

S. K. Deshpande is presently working as Scientist - $G$ in UGC-DAE Consortium for Scientific Research, Bhabha Atomic Research Center, Mumbai, Maharashtra, India. He has published over 90 publications with over 2000 citations in his research career. His research interest and expertise include Dielectric Spectroscopy, EXAFS, Perovskites.

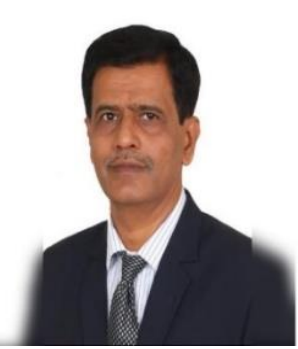

S. B. Kulkarni is currently working as Associate Professor in Department of Physics, The Institute of Science, 15, Madam Cama Road, Mumbai, Maharashtra, India. He has 25 years of experience in teaching and research. He has published over 65 publications with over 650 citations in his research career. His research interest includes multiferroic materials and energy storage devices.

Publisher's Note: Engineered Science Publisher remains neutral with regard to jurisdictional claims in published maps and institutional affiliations. 